\title{
Do feeding practices of obese dogs, before weight loss, affect the success of weight management?
}

\author{
Alexander J. German ${ }^{1 *}$, Shelley L. Holden ${ }^{1}$, Lucy J. Gernon ${ }^{1}$, Penelope J. Morris ${ }^{2}$ and Vincent Biourge ${ }^{3}$ \\ ${ }^{1}$ Department of Obesity and Endocrinology, Institute of Ageing and Chronic Disease, University of Liverpool, Leaburst \\ Campus, Chester High Road, Neston, Wirral CH64 7TE, UK \\ ${ }^{2}$ The Waltham Centre for Pet Nutrition, Waltham-on-the-Wolds, Melton Mowbray, Leicestershire, UK \\ ${ }^{3}$ Royal Canin Research Center, Aimargues, France \\ (Received 20 October 2010 - Revised 8 December 2010 - Accepted 10 January 2011)
}

\section{Abstract}

Dietary factors (e.g. feeding treats and table scraps) can predispose to obesity in dogs, but it is not known whether they also influence success of weight loss. Therefore, the aim of the present study was to determine which pre-weight-loss factors were associated with outcome of their weight management regimen in dogs. Information from ninety-five dogs attending the Royal Canin Weight Management Clinic, University of Liverpool (Wirral, UK), was reviewed. The effect of different food types (e.g. dry, wet and home-prepared), feeding practices (e.g. method of portion size calculation and number of meals per day) and use of treats was assessed on outcome measures of the weight management regimen. Before weight loss, most owners (sixty-three out of ninety-five, 66\%) fed twice daily, used complete dry food (seventy-two out of ninety-five, $76 \%$ ) and calculated portion size either by measuring cup (thirty-six out of ninety-five, $38 \%$ ) or by visual estimation (thirty-seven out of ninety-five, 39\%). Feeding treats was common and included purchased treats (forty-one out of ninety-five, $43 \%$ ), table scraps (twenty-four out of ninety-five, $25 \%$ ), pet food (eighty-three out of ninety-five, $87 \%$ ) and human food (eighty-one out of ninety-five, $85 \%$ ). The majority of feeding practices did not influence any outcome measure for the weight-loss period $(P>0.05$ for all). However, metabolisable energy intake during weight loss was significantly higher in dogs fed dry food $(P=0.047)$ and lower in dogs fed purchased snacks before weight loss $(P=0.036)$. Thus, most pre-weight-loss factors have limited effect on outcomes of weight loss. The significance of the associations identified between feeding of dried food and purchased treats, and weight-loss energy intake, requires further study.

Key words: Overweight: Canine: Nutrition

Obesity is a major medical concern in dogs and aetiology is usually multi-factorial $^{(1)}$. Previous studies have demonstrated associations between weight gain and a variety of dietary factors including the number of meals fed daily, feeding of table scraps and treats, the dog being present during preparation of food, and the quality of the brand of dog food $\mathrm{fed}^{(2-4)}$. Weight management strategies usually require the owner to make fundamental changes to their dog's lifestyle, including control of feeding practices. Therefore, it is possible that, in addition to predisposing to obesity, bad feeding practices could also influence the likelihood of success during a weight-loss regimen. In this respect, habits that are difficult to break could continue despite the advice of the veterinary professional, which may then affect the rate of weight loss or degree of dietary energy restriction. A previous human study identified a number of behavioural factors predictive of outcome of weight loss, including feeding behaviours such as emotional eating ${ }^{(5)}$. However, the authors are unaware of any similar study in companion animals. Nonetheless, previous studies have demonstrated that owner education and willingness to cooperate are essential for a weight-loss programme to succeed ${ }^{(6-8)}$. Therefore, the aim of the present study was to determine whether feeding habits, before weight loss, influenced a variety of outcomes in a cohort of dogs undergoing conventional weight management.

\section{Experimental methods}

Dogs

The study involved dogs referred to the Royal Canin Weight Management Clinic, University of Liverpool, Wirral, UK, for the investigation and management of obesity ${ }^{(9,10)}$, between March 2005 and January 2010. All were systemically well, and no significant abnormalities were observed on routine

Abbreviation: MER, maintenance energy requirement.

*Corresponding author: A. J. German, fax +44 151795 6101, email ajgerman@liv.ac.uk 
clinicopathological assessments ${ }^{(9)}$. The study adhered to the University of Liverpool Animal Ethics Guidelines and was approved by both the University of Liverpool Research Ethics Committee and the Waltham Ethical Review Committee. The owners of all participating animals gave their written informed consent.

\section{Feeding questionnaire}

Before the first appointment, owners were sent a questionnaire concerning current feeding practices. Owners completed this questionnaire and returned it either by post to the Royal Canin Weight Management Clinic or at the time of the appointment. During the first appointment, the weight management clinic nurse (S. L. H.) reviewed the answers provided by the owners, clarified any details that were unclear or illegible and gathered more information, where necessary.

Questions were asked about current and past feeding practices including the dog's main food intake, provision of fluids (i.e. water, milk and tea), feeding of additional food as treats and table scraps. This included the use of purchased treats and human foods.

\section{Weight-loss regimen}

All dogs in the study undertook a detailed weight management regimen, and full details have been described previously ${ }^{(9,10)}$. Briefly, at the initial consultation, patients were weighed and body composition was determined by fan-beam dual-energy X-ray absorptiometry (Lunar Prodigy Advance; GE Lunar, Madison, WI, USA) ${ }^{(11)}$. Composition results were used to calculate target weight, and an individually tailored weight management protocol was then instigated, using either a high-protein, high-fibre (Satiety Support; Royal Canin, Aimargues, France) or a high-protein, moderate-fibre (Obesity Management; Royal Canin) weight-loss $\operatorname{diet}^{(9,10)}$. For both diets, maintenance energy requirement (MER) was calculated using the estimate of target weight, and the initial allocation fed was individualised based upon sex and other factors (i.e. presence of associated diseases) ${ }^{(10)}$. In addition to dietary energy restriction, owners were counselled on the lifestyle and activity alterations ${ }^{(9)}$. All dogs were reassessed regularly during the weight-loss programme, and bodyweight measurements were taken and changes were made to the dietary plan, if necessary ${ }^{(9,10)}$. In addition, the weight management clinic nurse (S. L. H.) provided regular telephone support. A detailed re-evaluation was conducted after weight loss for dogs that successfully reached their target weight. Dogs were confirmed to have remained healthy based upon physical examination, routine haematological analysis, routine serum biochemical analysis and urinalysis. Body weight and body condition were recorded, and body composition was assessed by dual-energy X-ray absorptiometry.

\section{Statistical analysis}

Statistical analysis was performed with a computer software package (Stats Direct, version 2.6.8; Stats Direct Ltd.,
Altrincham, Cheshire, UK). The effect of different types of food (dry food, wet food, home-prepared food, etc.), feeding practices (method of weighing out food, number of meals per day, etc.) and use of additional food (purchased treats and human food) and fluids was assessed on various outcome measures including body fat percentage (measured by dualenergy X-ray absorptiometry), rate of weight loss during the regimen, percentage of weight lost during the regimen and the mean energy allocation required for weight loss. Continuous data were first assessed with the Shapiro-Wilk test and were confirmed to follow a normal distribution. Therefore, with the exception of Fisher's exact test (which was used to compare proportions), parametric tests were used, including two-sample $t$ tests and one-way ANOVA. The level of significance was set at $P<0.05$.

\section{Results}

\section{Study population}

During the period in question, 100 dogs were eligible for inclusion, and questionnaire details were obtained for ninety-five dogs, with a median age of 6 (range 1-14) years, and thirty-one different breeds were represented; Labrador Retriever (twenty-five), mixed breed (thirteen), Cavalier King Charles Spaniel (eight) and Golden Retriever (six) were most common, with the remaining breeds having between one and four individuals. Of the ninety-five dogs, fifty-two were male (forty-nine neutered) and forty-three were female (thirty-eight neutered).

\section{Summary of feeding practices before weight loss}

Main meal feeding. Feeding twice a day was most common (sixty-three out of ninety-five, 66\%), although some owners preferred feeding once daily (twenty-three out of ninety-five, $24 \%$ ) or three times daily (four out of ninety-five, $4 \%$ ), while food was left out all day in a small number of cases (five out of ninety-five, 5\%). Commercial complete dry food was given to seventy-two dogs (76\%), thirty-eight dogs ( $40 \%$ ) were fed commercial moist food and home-prepared food was given to twenty-two dogs (23\%). However, feeding more than one type of food was common (forty-one out of ninety-five, 43\%). When commercial food was used, the brand of food varied, with some dogs (twenty-two out of ninety-four, $23 \%$ ) being fed a mixture of brands. Of the ninety-five owners, thirty-seven (39\%) visually estimated the amount of food to give their dog (i.e. did not use either a feeding cup or weight scales), while thirty-six (38\%) used a measuring cup, sixteen (17\%) weighed food on kitchen scales and six used none of these methods.

\section{Fluid consumption}

All dogs were given water to drink, but five (5\%) were given tea and four ( $4 \%$ ) were given milk in addition to their food diet; one dog was given coffee three times a day, each with added milk and sugar. 


\section{Treats and table scraps}

Feeding treats was common and included purchased treats (forty-one out of ninety-five, $43 \%$ ) and table scraps (twentyfour out of ninety-five, $25 \%$ ), pet food (eighty-three out of ninety-five, $87 \%$ ) and human food (eighty-one out of ninety-five, $85 \%$ ). For the owners who admitted to feeding human food, a huge variety was reported, ranging from confectionary to fast food. Cheese (forty-four out of eightyone, $54 \%$ ), bread (forty-four out of eighty-one, 54\%), sweet biscuits (forty out of eighty-one, 49\%) and fruit (thirty-five out of eighty-one, $44 \%$ ) were particularly popular.

\section{Effect of feeding practices on outcome variables for weight loss}

Of the ninety-five dogs, forty-six (48\%) completed the weightloss programme and reached the target weight; the remaining forty-nine dogs (52\%) were either still enrolled but had not completed the programme, had been euthanised (for unrelated reasons) or had stopped prematurely (as decided by the owner). In the forty-six dogs that completed the programme, body fat percentage before weight loss was 42 (SD 5.9) $\%$, and this decreased to 28 (SD 6.6) \% after weight loss. Percentage of weight lost was 23 (SD 10.6) \% of starting body weight, and the mean rate of weight loss was 0.74 (SD 0.368 ) $\%$ per week. The mean energy allocation during weight loss was 58 (SD 6.4) \% of MER at target weight $\left(\text { MER }=440 \times \text { body weight }{ }^{0.75} \mathrm{~kJ} / \mathrm{d}\right)^{(12)}$.

For the forty-six dogs that completed the programme, preliminary analyses revealed that the weight-loss diet had no confounding effect on any of the results $(P>0 \cdot 1$ for all), and, therefore, dogs on the different diets were compared as a single group. The majority of food types, feeding practices and treat-giving practices had no significant effect on any of the outcome parameters (Table 1; $P>0.05$ for all). However, energy allocation required for weight loss was significantly higher in dogs fed dried food before weight loss (58 (SD 6.4)\% of MER at target weight) compared with those not fed dried food (53 (SD 6.1)\% of MER at target weight) $(P=0 \cdot 047)$. Energy allocation required for weight loss was also significantly lower in dogs fed purchased snacks (55 (SD 5.1)\% of MER at target weight) compared with those not fed these snacks (61 (SD 5.5)\% of MER at target weight $(P=0.036)$. Further analysis revealed that purchased snacks $(P=0 \cdot 004)$, but not table scraps or non-commercial treats $(P=0 \cdot 472)$, were more likely to be fed during weight loss to dogs that had received such snacks before weight loss than to dogs that had not received such snacks.

\section{Discussion}

The present study has demonstrated that the feeding practices of owners before weight loss have limited effect on the outcome of a conventional weight management regimen. This suggests that the education that clients receive during their weight management regimen is largely successful in changing habits and practices. These findings would be consistent with previous studies that have demonstrated the importance of owner education and willingness to cooperate during weight-loss programmes ${ }^{(6-8)}$. It also supports the finding of other studies that have suggested that an organised regimen improves success ${ }^{(13)}$.

However, the prior feeding of purchased treats was associated with the requirement for a lower energy allocation during weight loss but was not associated with overall percentage weight loss or rate of weight loss. Furthermore, such treats were more likely to be fed during weight loss in dogs that had received them before weight loss. The most likely explanation for these results is the fact that continued use of commercial treats was occasionally authorised during the weight-loss programme, and the allocation of the purpose-formulated weight-loss food reduced accordingly. A similar effect was not observed for table scraps and non-commercial treats, suggesting that owners were persuaded against feeding these rather than sanctioning their continued use. The prior use of dried food was associated with a high food allocation during weight loss, but with no significant effect on percentage weight lost or rate of weight loss. The reason for the effect of dried food is less clear and would require further study. A prospective study would be needed to determine whether this association was genuine, and what characteristic of weight loss may have conveyed the effect.

A striking finding of the present study was the variety of human food given as extras in this cohort of obese dogs before weight loss, which included a variety of vegetables, baked beans, poppadoms, prawn crackers, pasta, rice, sandwiches, yogurt, scrambled eggs, cooked meat and chocolate

Table 1. Effect of various feeding practices on outcomes in forty-six dogs completing the weight-loss programme*

\begin{tabular}{lccccccc}
\hline Variables & Meals/d $\neq$ & $\begin{array}{c}\text { Food } \\
\text { measuring } \dagger\end{array}$ & Moist $\ddagger$ & Dry $\ddagger$ & $\begin{array}{c}\text { Home- } \\
\text { prepared } \ddagger\end{array}$ & $\begin{array}{c}\text { Table } \\
\text { scraps } \ddagger\end{array}$ & $\begin{array}{c}\text { Purchased } \\
\text { snacks } \ddagger\end{array}$ \\
\hline Rate of weight loss & 0.207 & 0.716 & 0.382 & 0.513 & 0.226 & 0.833 & 0.694 \\
Percentage of weight loss & 0.732 & 0.261 & 0.853 & 0.390 & 0.688 & 0.958 & 0.268 \\
Weight-loss energy intake & 0.566 & 0.566 & 0.507 & $0.047 \S$ & 0.551 & 0.268 & $0.036 \|$ \\
\hline
\end{tabular}

* The results represent $P$ values for the statistical analyses performed.

† One-way ANOVA test was used.

$\ddagger$ Two-sample $t$ test was used.

$\S$ Higher energy allocation for weight loss in dogs fed dry food (58 (SD 6.4)\% of MER at target weight) than those not fed dry food (53 (SD 6.1 ) \% of MER at target weight).

|| Lower energy allocation for weight loss in dogs fed purchased snacks (55 (SD 5.1)\% of MER at target weight) than those not fed such snacks (61 (SD $5.5) \%$ of MER at target weight). 
(data not shown). These feeding habits probably reflect the eating habits of the owners and suggest that owners may not be giving proper consideration to the nutritional requirements of their dog when undertaking treat-giving behaviour ${ }^{(2)}$. Despite this, the present study found no association with the prior use of any of these treats and success of weight loss. Nonetheless, it was difficult to quantify the exact amounts given by owners, based upon their recollection, and a genuine effect might have been missed. Alternatively, the education delivered to owners during the weight management programme, teaching responsible treat-feeding, may have been successful in altering this behaviour.

The present study has a number of limitations that should be taken into account when interpreting the findings. First, the study population was taken from a weight management referral service and not from obesity clinics run in general practice. Given the fact that the clinic has a full-time veterinary nurse dedicated to weight management, it is possible that the education given to the owners (about altering feeding practices) and the level of support were greater than would normally be achieved. Thus, the level of effect of prior feeding behaviours may be greater on weight-loss programmes run in general practice. Second, the dogs in the present study were enrolled in a prospective weight-loss study, and this may have further biased the type of owners who were prepared to participate. A third concern is the fact that the study relied on information provided by the owners in questionnaires, and such an approach is prone to under-reporting in human studies $^{(14)}$. To minimise this effect, the weight management clinic nurse (S. L. H.) discussed the answers given in the questionnaire with owners after completion and clarified answers to verify accuracy. However, inaccurate reporting may still have been a factor.

In summary, the present study has demonstrated that prior feeding practices have limited effect on the outcome of weight management regimens in obese dogs. The significance of the associations identified between feeding of dried food and purchased treats, and weight-loss energy intake, requires further study.

\section{Acknowledgements}

The following conflicts of interest apply: Royal Canin manufactured the diets used in the present study; V. B. is an employee of Royal Canin; P. J. M. is an employee of Mars Petcare, Waltham-on-the-Wolds, UK; Royal Canin financially supports the posts of A. J. G. and S. L. H., at the University of Liverpool. The present study was funded by grants from
Royal Canin and Mars Petcare. The contributions of the authors are as follows: A. J. G. participated in the study design, data collection, analysis and interpretation of the data and writing of the manuscript; S. L. H. and L. J. G. were involved in the data collection, analysis and interpretation and reviewing the manuscript; P. J. M. and V. B. conducted the data analysis and interpretation, and helped in reviewing the manuscript.

\section{References}

1. German AJ (2006) The growing problem of obesity in dogs and cats. J Nutr 136, 1940S-1946S.

2. Kienzle E, Bergler R \& Mandernach A (1998) A comparison of the feeding behavior and the human-animal relationship in owners of normal and obese dogs. J Nutr 128, 2779S-2782S.

3. Robertson ID (2003) The association of exercise, diet and other factors with owner-perceived obesity in privatelyowned dogs from Metropolitan Perth, WA. Prev Vet Med 58, 75-83.

4. Bland IM, Guthrie-Jones A, Taylor RD, et al. (2009) Dog obesity: owner attitudes and behaviour. Prev Vet Med $\mathbf{9 2}$, 333-340.

5. Leon GR \& Sternberg Rosenthal B (1984) Prognostic indicators of success or relapse in weight reduction. Int $J$ Eat Disord 3, 15-24.

6. Sloth C (1992) Practical management of obesity in dogs and cats. J Small Anim Pract 33, 178-182.

7. Laflamme DP (2006) Understanding and managing obesity in dogs and cats. Vet Clin North Am Small Anim Pract 36, $1283-1295$.

8. Roudebush P, Schoenherr WD \& Delaney SJ (2008) An evidence-based review of the use of therapeutic foods, owner education, exercise, and drugs for the management of obese and overweight pets. J Am Vet Med Assoc 233, $717-725$.

9. German AJ, Holden SL, Bissot T, et al. (2007) Dietary energy restriction and successful weight loss in obese client-owned dogs. J Vet Intern Med 21, 1174-1180.

10. German AJ, Holden SL, Bissot T, et al. (2010) A high protein high fibre diet improves weight loss in obese dogs. Vet J 183, 294-297.

11. Raffan E, Holden SL, Cullingham F, et al. (2006) Standardized positioning is essential for precise determination of body composition using dual-energy X-ray absorptiometry in dogs. J Nutr 136, 1976S-1978S.

12. National Research Council (NRC) (2006) Nutrient Requirements of Dogs and Cats. Washington, DC: National Academy Press.

13. Yaissle JE, Holloway C \& Buffington CAT (2004) Evaluation of owner education as a component of obesity treatment programs for dogs. J Am Vet Med Assoc 224, 1932-1935.

14. Heitmann BL, Lissner L \& Osler M (2000) Do we eat less fat, or just report so? Int J Obes 24, 435-442. 